\title{
DESENVOLVIMENTO DE UM FRAMEWORK PARA O PLANEJAMENTO DE COMPRAS PÚBLICAS: ESTUDO EM UMA UNIVERSIDADE FEDERAL
}

\author{
DEVELOPING A FRAMEWORK FOR PUBLIC PROCUREMENT PLANNING: \\ STUDY AT A FEDERAL UNIVERSITY
}

\author{
Edjane Cortez da Cruz \\ Mestre em Gestão de Processos Institucionais \\ Universidade Federal do Rio Grande do Norte - UFRN \\ Natal, Rio Grande do Norte - Brasil \\ edjanecortezc@gmail.com \\ Thiago de Oliveira \\ Graduado em Administração \\ Universidade Federal do Rio Grande do Norte - UFRN \\ Parnamirim, Rio Grande do Norte - Brasil \\ thiago.oliveira.adm@outlook.com \\ André Morais Gurgel \\ Doutor em Engenharia de Produção \\ Universidade Federal do Rio Grande do Norte - UFRN \\ Natal, Rio Grande do Norte - Brasil \\ andmgurgel@gmail.com
}

\section{Resumo}

As compras públicas materializam as políticas governamentais e promovem o desenvolvimento econômico e sustentável das instituições e da sociedade. Em parte dos órgãos públicos brasileiros, a burocracia do processo licitatório, somada à limitação de recursos, dificulta a execução e favorece a morosidade. Nesse contexto, é relevante a aplicação de alternativas que visam melhorar a estrutura do processo de compras, atingir resultados estratégicos, evitar retrabalho e desperdícios nas organizações. Assim, a presente pesquisa objetiva apresentar o desenvolvimento de um framework para planejamento de compras públicas alinhado às metodologias da gestão de projetos, como foco nas universidades federais. Para isto, foi construído um estudo de caso qualitativo e aplicado, utilizando-se a abordagem da pesquisa documental para averiguação dos normativos e adaptação das técnicas de gestão de projetos; da observação participante e workshop para validação do framework; e a análise de conteúdo para a avaliação dos dados coletados. Os resultados sugerem que a metodologia sistematizada da proposta tornou a atuação da equipe mais dinâmica e contribuiu para que as entregas fossem concluídas dentro do prazo, conforme os critérios e escopo planejados. Do ponto de vista teórico, o trabalho enriquece a literatura sobre o uso e o impacto do gerenciamento de projetos, sobretudo na construção de novas ferramentas pautadas nessa abordagem, ao demonstrar a sua aplicabilidade no setor público brasileiro.

Palavras-chave: Compras públicas. Gestão de projetos. Framework.

\begin{abstract}
Public procurement materializes government policies and promotes the sustainable development of institutions and society. In Brazilian public agencies, the bureaucracy of the purchasing process and the limited resources difficult the process execution. In this context, it is relevant to apply alternatives that aim to improve the structure of the purchasing process, achieve strategic results, avoid rework and waste in the associations. Thus, this research aims to present the development of a framework for planning public procurement in line with project management methodologies focused on federal universities. For this, a qualitative and applied case study was built, using the documentary research approach to ascertain the norms and adapt the project management techniques. We used participant observation and workshops to validate the framework. Then, we used content analysis to evaluate the collected data. Thus, we observed that the results obtained with the systematized methodology became the performance of the team more dynamic and contributed to complete the projects within the deadline, according to the planned criteria and scope. From a theoretical point of view, the work enriches the
\end{abstract}


literature on the use and impact of project management, especially in the construction of new tools based on this approach, by demonstrating its applicability in the Brazilian public sector.

Keywords: Public procurement. Project management. Framework.

\section{Cite como - American Psychological Association (APA)}

Cruz, E. C., Oliveira, T., \& Gurgel, A. M. (2020, set./dez). Desenvolvimento de um framework para o planejamento de compras públicas: estudo em uma Universidade Federal. Revista de Gestão e Projetos (GeP), 11(3), 94-116. https://doi.org/10.5585/gep.v11i3.18475.

\section{Introdução}

Planejar é fundamental, tanto na esfera privada como no setor público. Para este último, a importância se amplia, uma vez que o seu principal objetivo é atender as demandas da sociedade (Matias-Pereira, 2012). As políticas públicas, por sua vez, são viabilizadas por meio das compras públicas que, além de fornecerem os suprimentos, se configuram como instrumento de execução orçamentária (Ferrer, 2015; Santana, 2015; Fernandes, 2019).

A gestão pública brasileira é norteada pelo Plano Plurianual (PPA), a Lei de Diretrizes Orçamentárias (LDO) e a Lei Orçamentária Anual (LOA), instrumentos que vinculam o planejamento à execução do dinheiro público (Matias-Pereira, 2012; Costa, Braga \& Andrioli, 2017; Fenili, 2018). Com os recursos orçamentários cada vez mais escassos, cuja Emenda Constitucional do Teto dos Gastos Públicos (EC 95/2016) é uma referência, a aplicação e qualidade do gasto são fatores determinantes à sustentabilidade dos órgãos (Ramírez-Gutiérrez, Barrachina-Palanca \& Ripoll-Feliu, 2020; Campos, Dantas, Milito\& Silva, 2020).

\section{A Organização para a Cooperação e} Desenvolvimento Econômico (OCDE) aponta que as aquisições governamentais correspondem há aproximadamente $13 \%$ do Produto Interno Bruto (PIB) brasileiro (Santana, 2015), mantendo média similar em outros países (Fuentes-Bargues, FerrerGisbert\&González-Cruz， 2018). Conforme dados do Painel de Compras do Governo Federal, as compras públicas ${ }^{1}$ movimentaram aproximadamente 48 bilhões de reais em 2018, incluídas todas as modalidades de licitação. Isto revela o poder de compra do Estado à medida que as Instituições Federais de Ensino Superior (IFES) também despontam como grandes unidades compradoras (Ramírez-Gutiérrez, Barrachina-Palanca \& Ripoll-Feliu, 2020).

No contexto das IFES, as universidades federais são classificadas como autarquias especiais (Justen Filho, 2016) em razão de sua autonomia gerencial. No entanto, como entidades públicas, necessitam alinhar os seus processos institucionais à legislação correlata para atender aos normativos expedidos por

\footnotetext{
${ }^{1}$ Neste artigo, os termos compras públicas, compras federais, contratações públicas, aquisições públicas, aquisições governamentais e suprimentos governamentais são considerados sinônimos.
} 
ministérios e pelo Tribunal de Contas da União (TCU).

No âmbito organizacional, as ações podem ser executadas por meio de projetos (Veras, 2016), que são caracterizados por sua temporalidade e singularidade (Carvalho \& Rabechini Júnior,2017), além de uma oportunidade para solucionar problemas e implantar melhorias (Vargas, 2009, 2018), disseminar ou alterar estratégias e fomentar o alcance dos objetivos estratégicos e institucionais (PMI, 2017; Campos et al., 2020; Link, Silva, Barichello \& Dal Magro, 2020; Oliveira, Oliveira \& Michaloski, 2020).

A gestão de projetos apresenta o conhecimento sistematizado e uso de ferramentas e metodologias próprias para alcançar os objetivos estratégicos (Kerzner, 2006; Veras, 2016; Carvalho \& Rabechini Júnior, 2017; PMI, 2017). No ciclo de vida do projeto, há os grupos de processos de gerenciamento (Medeiros, 2017), que correspondem à iniciação, planejamento, execução, monitoramento e controle e encerramento (PMI, 2017). De modo correspondente, o planejamento de compras públicas também é dividido em etapas, as quais exigem documentos específicos para o processo licitatório (Santana, 2015; Fenili, 2018).

Dadas as similaridades, o gerenciamento de projetos pode ser um aliado no processo de planejamento de compras, uma vez que também obedece a uma sequência lógica, busca eficiência e apresentar resultados, consoante os objetivos propostos, por meio de entregas e do produto final (Veras, 2016; Carvalho \& Rabechini Júnior, 2017; Carvalho $\&$ Nelson, 2020).

Portanto, a partir da inter-relação entre essas duas temáticas, surge a seguinte questão de pesquisa: como uma metodologia estruturada no ciclo de vida do gerenciamento do projeto pode contribuir para o planejamento de compras públicas? Considerando a problemática, o objetivo deste trabalho é apresentar uma proposta de framework alinhado às metodologias de gestão de projetos para o planejamento das compras públicas nas universidades federais.

$\mathrm{Na}$ fase interna ocorre a maioria das falhas associadas ao insucesso da licitação, como inconsistência nas especificações do objeto e na pesquisa de preços (Carvalho \& Nelson, 2020; Neves \& Moré, 2020). Esses fatores conduzem ao cancelamento e, consequentemente, a indisponibilidade de itens no catálogo, podendo comprometer a qualidade e a continuidade dos serviços na instituição (Avigo, Joao-Roland, 2019; Soares, CorreiaNeto, Ribeiro \& Ferreira, 2019). Apesar disso, parte da literatura tem se dedicado mais ao aspecto jurídico do que ao planejamento e à gestão das compras públicas (Santana, 2015; Fenili, 2018; Fernandes, 2019).

\section{Planejamento de compras públicas}

As compras públicas compreendem as contratações de bens e serviços realizados mediante os critérios estabelecidos pela legislação que disciplina a matéria, com 
observância ao preço econômico, qualidade e celeridade, visando suprir os órgãos durante o desempenho de suas atividades-fim (Ferrer, 2015; Santana, 2015; Fenili, 2018). Compõem um processo sistêmico e transversal, dividido nas fases preparatória, transacional, contratual e gestão dos fornecedores. Posteriormente, os dados gerados permitem retroalimentar o ciclo e auxiliar o gestor na tomada de decisão (Fernandes, 2019; Araújo, Matos \& Ensslin, 2020).

O planejamento das compras e contratações integra a fase interna do processo licitatório, da qual resultam os Estudos Técnicos Preliminares e Termo de Referência ou Projeto Básico, instrumentos que contêm os requisitos e especificações do objeto a ser adquirido (Camarão \& Chrispim, 2016; Costa, Braga \&Andrioli, 2017). Agrega a identificação das demandas futuras e dos processos prioritários da gestão mediante sua vinculação ao orçamento. Assim, o Plano Anual de Compras (PAC) materializa a programação de compras das instituições, estabelece o cronograma de execução e os indicadores de avaliação e desempenho (Araújo, Matos \& Ensslin,2020). Por esses aspectos, o instrumento é considerado uma ferramenta de governança (Fenili, 2018).

A etapa do planejamento é determinante pois concentra muitos problemas relacionados às falhas nas licitações, tais como itens mal especificados e pesquisa de preços incompatível com os valores praticados pelo mercado fornecedor. Isto reflete em retrabalho, morosidade, indisponibilidade de materiais e serviços e desperdício de recursos, entre outros (Soares et al., 2019; Carvalho \& Nelson, 2020; Neves \& Moré, 2020).

Por outro lado, alguns fatores contribuem para o sucesso do processo licitatório, dos quais: pesquisa de preços compatível com os valores de mercado (Fenili, 2018); Estudos Técnicos bem elaborados (Costa, Braga \& Andrioli, 2017); objetos devidamente especificados no Termo de Referência (Neves \& Moré, 2020); calendário anual para coleta de demandas (Fenili, 2018; Nascimento \& Santos, 2018); histórico sobre a qualidade do produto e cumprimento dos prazos de entrega (Fenili, 2018; Soares et al. 2019; Araújo, Matos \& Ensslin, 2020; Campos et $a l ., 2020)$.

Em decorrência disso, nos últimos anos o Governo Federal, com o apoio do Ministério do Planejamento e do TCU, tem promovido ações inovadoras ao processo de compras, as quais demandam adequação dos processos institucionais. Nesse contexto, considerando os diferentes níveis de maturidade dos órgãos, as instruções normativas têm a finalidade de interpretar o que já está previsto no texto legal, porém de forma mais detalhada; funcionam como uma espécie de metodologia, mas com caráter impositivo (Bittencourt, 2015; Neves \& Moré, 2020).

Diferente de outras entidades com atribuições mais reduzidas, as universidades federais, em razão dos seus processos de ensino, pesquisa e extensão, necessitam de 
variados serviços e materiais (insumos, equipamentos, transporte, serviços terceirizados, publicação de artigos), considerados como atividades-meio que dão suporte às atividades-fim (Fenili, 2018). Desse modo, inconsistências nos processos de compras podem impactar negativamente a qualidade dos serviços prestados à comunidade universitária ou comprometer a sua continuidade (Santana, Camarão \& Chrispim, 2016, Nascimento \& Santos, 2018; Soares et al.,2019).

\section{Gestão de projetos}

Projeto é o conjunto de atividades temporárias, com responsabilidade de execução definida, objetivos, escopo, prazo, recursos específicos, orientado a resultados e à criação de um novo produto ou serviço; conta com a participação de clientes, patrocinadores e stakeholders, além da equipe designada para esse fim (Kerzner, 2006; Veras, 2016; Carvalho \& Rabechini Júnior, 2017; PMI, 2017; Vargas, 2018).

Nessa perspectiva, gerenciar projetos se relaciona ao uso de ferramentas que reúnem o conhecimento, a capacidade técnica (Oliveira, Oliveira \& Michaloski, 2020) e possibilitam controlar e monitorar o todo o ciclo de vida. Este é dividido em fases para melhor estruturar a sua gestão (Kerzner, 2006; Vargas, 2018; Veras, 2016; PMI, 2017). Dadas as características, pode contribuir tanto para organizações privadas quanto para instituições públicas, no que concerne a estruturar a mudança, resolver os problemas, otimizar os recursos organizacionais e implementar inovações (Medeiros, 2017; Link et al., 2020).

Conforme exposto no $\mathrm{PMBOK}^{\circledR}$, o ciclo de vida do projeto é composto por início, organização e preparação, execução do trabalho e encerramento do projeto (Carvalho \& Rabechini Júnior, 2017). Quanto aos tipos, o PMI (2017) indica que os ciclos de vida podem ser preditivos, iterativos, adaptativos ou híbridos.

Para o gerenciamento de cada uma das fases do ciclo de vida, há os grupos de processos (iniciação, planejamento, execução, monitoramento e controle e encerramento), cujas atividades são alimentadas pelas dez áreas do conhecimento (integração, escopo, cronograma, custos, qualidade, recursos, comunicações, riscos, aquisições e partes interessadas). Por sua vez, esses grupos de processos geram entradas ou saídas que caracterizam a conclusão de uma fase ou etapa (Vargas, 2018). Tal exemplo é o Termo de Abertura do Projeto (TAP), que marca a conclusão da iniciação e serve como input à etapa do planejamento. (Kerzner, 2006; Vargas, 2018; Veras, 2016; PMI, 2017).

No contexto da administração pública moderna, mais orientada ao uso de soluções tecnológicas para inovar seus processos, o gerenciamento de projetos constitui uma ferramenta estratégica para executar as mudanças necessárias (Medeiros, 2017; Carvalho \& Nelson, 2020). Além disso, "alinhar os projetos e os processos com os 
objetivos da organização" (Veras, 2016, p.9) também pode configurar uma forma mais adequada de aplicação dos recursos financeiros.

A gestão de projetos proporciona benefícios para as organizações públicas, dentre os quais: sustentabilidade organizacional, inovação nos produtos e serviços entregues à sociedade, gestão por resultados, liderança, adequada gestão do orçamento, melhor comunicação, avaliação e gestão das equipes de trabalho (Carvalho \& Nelson, 2020; Link et al., 2020).

\section{Modelos visuais em gerenciamento de projetos}

Diante da complexidade organizacional, as boas práticas indicadas pelo gerenciamento tradicional de projetos, que são mais burocráticas e exigem um bom nível de maturidade, já não atendem plenamente ao ritmo dinâmico, simples e integrado dos processos atuais (Veras, 2016; Medeiros, 2017).

Nessa perspectiva, a adoção de novas técnicas se faz necessária (Oliveira, Oliveira \& Michaloski, 2020) para que as lacunas deixadas pelos métodos tradicionais sejam preenchidas por modelos visuais como canvas e kanban, quadros utilizados para apoiar a execução visual de tarefas de projetos e processos (Medeiros et al.,2017). Se usados adequadamente, eles despontam como um método eficiente para melhorar processos, bem como introduzir e gerenciar mudanças nas organizações (Eaidgah, Maki, Kurczewski \& Abdeekhodae,2016).
Seguindo essa tendência, Finocchio Júnior (2013), inspirado no Business Model Generation (BMG) de Alexander Osterwalder e Yves Pigneur, criou o Project Model Canvas (PMC), cuja aplicação é focada no plano do projeto. Por seu turno, buscando corrigir inconsistências identificadas nessas ferramentas visuais, Veras (2016) desenvolveu o Life Cycle Canvas ${ }^{\circledR}$ (LCC), um modelo que abrange todo o ciclo de vida do projeto. Embora inspirado no BMG e o PMC, esse artefato se propõe mais completo, pois é adaptável a vários contextos e conjuga todas as fases do projeto em uma única tela (Medeiros, Araújo \& Oliveira, 2018).

O LCC também é apoiado na abordagem $5 \mathrm{~W} 2 \mathrm{H}$ e alinhado às recomendações do $\mathrm{PMBOK}^{\circledR}$. Além disso, as telas são ajustadas ao longo do ciclo de vida do projeto, destinando-se às etapas de iniciação, planejamento, execução e monitoramento e controle e encerramento (Veras, 2016; Medeiros, 2017; Medeiros et al.,2017). Para Medeiros, Gurgel \& Veras (2017), em conjunto com o kanban, a gestão de projetos baseada nesse modelo visual auxilia no controle, execução e integração das partes interessadas, além de facilitar a comunicação (Medeiros et al., 2017; Barbosa, França \& Parreiras, 2018; Oliveira, Oliveira \& Michaloski, 2020) e a gestão do ciclo de vida; enquanto o quadro de tarefas contribui para o engajamento da equipe.

$\mathrm{O}$ referencial de literatura fundamenta a base teórica que conecta os temas planejamento de compras públicas e gerenciamento de projetos, com ênfase nos modelos visuais. $\mathrm{Na}$ 
mesma medida, o construto alicerça as discussões dos resultados obtidos neste estudo e oferece suporte às contribuições acadêmicas.

\section{Metodologia}

Esta pesquisa é caracterizada como estudo de caso (Yin, 2001), uma vez que investiga o fenômeno em determinado contexto e verifica o problema in loco (Flick, 2013). Por seu recorte ser restrito a um grupo específico, qual seja uma equipe de planejamento de compras de uma universidade federal, é classificada como pesquisa de campo. Nessa abordagem, o pesquisador se dispõe a averiguar a relação entre fenômenos, os quais exigem maior observação (Prodanov \& Freitas, 2013).

$\mathrm{O}$ estudo recorre à abordagem qualitativa, pois visa compreender a atuação de um grupo ou uma organização. Esse tipo de enfoque é flexível quanto à introdução de novas perguntas e questões durante e até após o andamento da pesquisa. Além disso, permite fazer alterações no processo de interpretação dos fatos, haja vista que se propõe analisar e interpretar o mundo a partir de representações (Sampieri, Collado \& Lucio, 2013).

Para fins deste estudo de caso, e visando atender aos objetivos propostos, optouse pela triangulação dos seguintes instrumentos de coleta de dados: a pesquisa documental, a observação participante e o workshop. Quanto às etapas, o estudo foi dividido em inicial, desenvolvimento e produto.

A pesquisa documental consiste em interpretar dados que não foram necessariamente produzidos pela e para pesquisa em questão, mas que podem fomentála (Flick, 2013). Diante disso, foram consultados normativos e dados consoantes a sua correspondência com o tema investigado. Outrossim, os documentos e artefatos resultantes da aplicação do framework também foram passíveis de análise.

No primeiro momento, foi realizada a consulta das Instruções Normativas (IN) no site de compras governamentais, o Comprasnet, que reúne a matéria sobre a área de contratações públicas brasileiras. Em seguida, foram estabelecidos como critérios de escolha para análise mais aprofundada: i) manter relação direta com o tema planejamento de compras públicas, ii) focar nas etapas e procedimentos da contratação; iii) ser destinada à contratação de serviços comuns; e iv) ter sido publicada entre os anos de 2008 a 2018.

Como resultado, as IN 5/2017 e IN 1/2018, ambas expedidas pelo Ministério do Planejamento, constituíram o corpus da análise, visto conjugarem as etapas do planejamento da contratação, seus atores, designações e orientações para elaboração dos documentos, além de estabelecerem o fluxo processual (Fenili, 2018). Quanto ao ciclo de vida do gerenciamento do projeto, a investigação foi pautada na literatura correspondente ao tema.

Para averiguar a conexão entre as duas áreas, buscou-se observar aspectos inerentes a ambas, pois esta pesquisa concebe o planejamento de compras como um projeto. Desse modo, foram considerados critérios de 
análise: iniciação, objetivo, singularidade, temporalidade, sequência, prazos, pessoas e conhecimento; mantendo-se, assim, alinhamento com o observado nos estudos de Vargas (2009, 2018), PMI (2017), Carvalho \& Rabechini Júnior (2017), bem como respeitada a nomenclatura do gerenciamento de projetos.

$$
\text { Ministrado pelos próprios }
$$
pesquisadores, o workshop denominado "Framework Planejamento da Contratação aplicado à Equipe de Planejamento" foi apresentado à equipe de planejamento em 18 de dezembro de 2018, das $9 \mathrm{~h}$ às $11 \mathrm{~h}$. Teve como objetivo estruturar a aplicação do modelo, bem como simulá-lo em situações reais do ambiente de trabalho. Após a explanação da metodologia, partiu-se para imersão e preenchimento do Canvas do Planejamento da Contratação, uma das atividades que integram a etapa de Iniciação, a ser detalhada no tópico Resultados.

Por se tratar de uma pesquisa de aplicação prática, a técnica de observação participante pretendeu, além da imersão dos pesquisadores, investigar a adaptação da equipe à nova metodologia de trabalho proposta pelo framework. Com essa medida, foi possível verificar a contribuição efetiva da proposta para a conclusão das entregas estabelecidas. Outro ponto foi avaliar a comunicação e troca de informações entre os participantes, seu comprometimento e atuação. Essa experiência possibilitou fazer melhorias na versão final do modelo, a partir o ponto de vista dos sujeitos. Quanto aos critérios a serem observados na etapa de validação, tem-se: adaptação, participação, comunicação e contribuições práticas.

Dos quatro encontros presenciais ocorridos entre dezembro de 2018 e janeiro de 2019, três foram registrados em áudio, com a devida anuência dos participantes e para uso exclusivo desta pesquisa. A técnica possibilitou a integração com o ambiente e o problema de pesquisa, a fim de analisar e interpretar o recorte observado (Sampieri, Collado \& Lucio, 2013).

Ao final do último encontro foram registradas as lições aprendidas, com o objetivo de avaliar a percepção da equipe sobre a metodologia aplicada. A técnica também consistiu um procedimento de validação, estruturado com base nas boas práticas do PMBOK $^{\circledR}$ e autores da área (Veronese, 2014; Chaves, Araújo, Teixeira \& Virginio, 2016; Duffield \& Whitty, 2016).

O tratamento dos dados compreendeu a compilação das informações, seguida da separação dos assuntos por área temática. Para Flick (2013) consiste em registrar, transcrever e comparar as informações para interpretá-las. Ademais, é comum em pesquisas qualitativas o pesquisador ter maior flexibilidade para, já no decorrer do estudo, estabelecer conexões e optar por dados que mais agregam valor aos aspectos desejados (Sampieri, Collado \& Lucio, 2013).

As gravações dos áudios totalizaram 171 minutos, dos quais resultaram 21 páginas escritas. De posse dos trechos das conversas, aliada às anotações particulares, estabeleceu-se 
a correspondência entre os dados e os objetivos da pesquisa. Em seguida, foi feita a comparação entre os dados obtidos e os critérios determinados para validação do framework.

Para a análise dos dados foi utilizada a Análise de Conteúdo. Esta ocorreu mediante a organização e transcrição das informações coletadas, para posterior seleção de trechos e interpretação, o que possibilitou o levantamento de inferências ou explicações acerca do fenômeno estudado (Sampieri, Collado \& Lucio, 2013). A leitura das conversas permitiu manter conexões entre o tema de pesquisa, correlacionando a abordagem teórica à prática administrativa.

No que concerne aos aspectos éticos, salienta-se que o acesso e a utilização dos dados ocorreram mediante a comunicação e autorização prévia das unidades e sujeitos da pesquisa, que preencheram o Termo de Consentimento de Gravação de Voz. No tópico a seguir, serão apresentados os resultados, a análise e as discussões.

\section{Resultados}

A Instrução Normativa $n^{\circ} 5$, de 26 de maio de 2017, da Secretaria de Gestão do Ministério do Planejamento, Desenvolvimento e Gestão dispõe sobre as regras e diretrizes do procedimento de contratação de serviços sob o regime de execução indireta no âmbito da Administração Pública federal direta, autárquica e fundacional. $\mathrm{O}$ art.19 indica como fases: planejamento da contratação, seleção do fornecedor e gestão do contrato. No tocante ao planejamento da contratação, a redação do art. 20 prevê as seguintes etapas: I - Estudos preliminares, II - Gerenciamento de Riscos e III - Termo de Referência ou Projeto Básico.

O normativo também detalha os procedimentos iniciais do planejamento, que consistem na elaboração do documento para formalização da demanda. Tal instrumento deve conter: a) justificativa da necessidade da contratação; b) quantidade do serviço a ser contratado; c) previsão para o início da prestação do serviço e d) indicação de um ou mais servidores para compor equipe que elaborará os estudos preliminares e o mapa de gerenciamento de risco.

A etapa do planejamento está inserida na fase preparatória da licitação. Desse modo, consoante ao que postula a IN 5/2017 em seu artigo 20, os referidos documentos devem expressar a necessidade da compra. Assim, a justificativa do gestor de compras para a aquisição pretendida deve responder a questões como por que, para que, para quem, como e quando sobre o objeto a ser contratado (Santana, Camarão \& Chrispim, 2016).

Uma vez que esta pesquisa considera o planejamento de compras públicas como um projeto, fez-se necessário estabelecer, portanto, a conexão entre as respectivas áreas, conforme atendimento aos seguintes critérios: iniciação, objetivo, singularidade, temporalidade, sequência, prazos, pessoas e conhecimento (Vargas, 2009; PMI, 2017; Carvalho \& Rabechini Júnior, 2017). 
Os dados do estudo sugerem que são comuns tanto às etapas do planejamento da contratação quanto às fases do ciclo de vida do gerenciamento do projeto a temporalidade e as atividades sequenciadas. Além disso, os projetos são executados por pessoas que assumem papéis e responsabilidades definidos e atuam em prol de cumprimento dos objetivos traçados dentro do tempo previsto (Vargas, 2009; Carvalho \& Nelson, 2020).

Para Vargas (2009), os projetos são iniciados para solucionar algum problema ou implementar melhorias no ambiente organizacional. Já o planejamento das compras é necessário quando uma determinada demanda é identificada (Ferrer, 2015; Santana, 2015).

Os projetos também objetivam o cumprimento de metas organizacionais e são aliados nos processos de inovação (Carvalho \& Rabechini Júnior, 2017; Campos et al., 2020). Da mesma maneira, o planejamento de compras (Carvalho \& Nelson, 2020), sob a perspectiva do PAC, tem como objetivo o preenchimento de informações alusivas à contratação pública (Fenili, 2018; Nascimento \& Santos, 2018; Neves \& Moré, 2020).

Os temas resguardam características singulares. No PAC, cada demanda requer um planejamento específico, visto que as condições, soluções oferecidas pelo mercado, orçamento disponível e prioridades podem variar de acordo com cada instituição (Nascimento \& Santos, 2018; Avigo \& JoãoRoland, 2019; Soares et al.,2019). Os projetos também são únicos, sendo este um traço que os distingue das atividades rotineiras (Carvalho \& Rabechini Júnior, 2017).

Ainda nessa perspectiva, cabe destacar que tanto o planejamento de compras quanto o ciclo de vida do gerenciamento do projeto são executados dentro de um prazo. Enquanto o primeiro deve estar alinhado ao calendário de compras institucional, o segundo pode variar de acordo com a complexidade de cada iniciativa (Soares et al.,2019; Campos et at., 2020).

Por fim, tanto os projetos como o planejamento de compras públicas são executados por pessoas (Kerzner, 2006; PMI, 2017) dentro do ambiente organizacional. Em cada uma das respectivas áreas, a definição de papéis e responsabilidades está associado à função que o membro desempenha na equipe (Carvalho \& Rabechini Júnior,2017; Araújo, Matos \& Ensslin, 2020), considerando também seu conhecimento e expertise relacionado à área de interesse (Vargas, 2018). A Tabela 1 sintetiza a relação entre as etapas do planejamento da contratação e o ciclo de vida do gerenciamento do projeto: 
Tabela 1 - A conexão entre o ciclo de vida do projeto e o planejamento de compras

\begin{tabular}{|c|c|c|}
\hline CARACTERÍSTICAS & $\begin{array}{l}\text { CICLO DE VIDA DO } \\
\text { GERENCIAMENTO DO } \\
\text { PROJETO }\end{array}$ & $\begin{array}{c}\text { PLANEJAMENTO DE COMPRAS } \\
\text { PÚBLICAS }\end{array}$ \\
\hline INICIAÇÃO & $\begin{array}{l}\text { Processos utilizados para definir uma } \\
\text { nova fase ou novo projeto; } \\
\text { autorização para início do projeto }\end{array}$ & $\begin{array}{l}\text { Identificação da demanda, das partes } \\
\text { interessadas e a equipe de } \\
\text { planejamento; elaboração de TAP }\end{array}$ \\
\hline PLANEJAMENTO & $\begin{array}{l}\text { Processos realizados para definir as } \\
\text { entregas, cronograma, atividades, } \\
\text { custos, responsáveis e as ações a } \\
\text { serem executadas }\end{array}$ & $\begin{array}{l}\text { Definição das atividades, por meio de } \\
\text { entregas menores; designação de } \\
\text { responsáveis; análise do mercado } \\
\text { fornecedor e das soluções disponíveis; } \\
\text { pesquisa de preço }\end{array}$ \\
\hline EXECUÇÃO & $\begin{array}{l}\text { Processos utilizados para concluir as } \\
\text { atividades planejadas, conforme os } \\
\text { critérios estabelecidos }\end{array}$ & $\begin{array}{l}\text { Elaboração dos documentos: } \\
\text { Documento de Oficialização da } \\
\text { Demanda (DOD), Estudos Técnicos } \\
\text { (ET) e Mapa de Riscos (MR), } \\
\text { conforme os critérios de qualidade } \\
\text { estabelecidos nos normativos, e prazo } \\
\text { do calendário institucional. }\end{array}$ \\
\hline $\begin{array}{l}\text { MONITORAMENTO E } \\
\text { CONTROLE }\end{array}$ & $\begin{array}{l}\text { Processos utilizados para monitorar, } \\
\text { controlar e acompanhar o andamento } \\
\text { do projeto, bem como proceder as } \\
\text { mudanças necessárias durante o ciclo } \\
\text { de vida. }\end{array}$ & $\begin{array}{l}\text { Observância ao cumprimento dos } \\
\text { prazos e do calendário de compras; } \\
\text { gerenciamento das mudanças; } \\
\text { verificação da anuência da gestão para } \\
\text { o prosseguimento do trâmite processual }\end{array}$ \\
\hline ENCERRAMENTO & $\begin{array}{l}\text { Processos utilizados para concluir } \\
\text { formalmente o projeto. }\end{array}$ & $\begin{array}{l}\text { Todos os documentos elaborados e } \\
\text { validados pela equipe e pelo } \\
\text { patrocinador; informações para o } \\
\text { preenchimento do PAC }\end{array}$ \\
\hline
\end{tabular}

Fonte: Dados da pesquisa (2019).

Identificada a conexão entre o ciclo de vida do projeto e planejamento de compras públicas, foi desenvolvido um artefato tipo canvas para auxiliar as equipes de planejamento da contratação a pensarem soluções em seu contexto de trabalho (Fernandes, 2019; Soares et al.,2019). Dessa forma, a ferramenta associa a filosofia do $5 \mathrm{~W} 2 \mathrm{H}$ a um quadro visual (Finocchio Júnior, 2013; Veras, 2016) para facilitar a comunicação e troca de informações entre os participantes (Tjell \& Bosch-Sijtsema, 2015; Oliveira, Oliveira, \& Michaloski, 2020).
Por seu turno, a ferramenta está alinhada à IN 5/2017, pois estimula os participantes a responderem, ainda que de forma sintética, as perguntas constantes no Documento de Oficialização da Demanda (DOD), nos Estudos Técnicos (ET) e no Mapa de Riscos (MR). A Figura 1 apresenta o Canvas do Planejamento da Contratação, cuja nomenclatura será reduzida para Canvas nas próximas menções. 
Figura 1 - Canvas Planejamento da Contratação

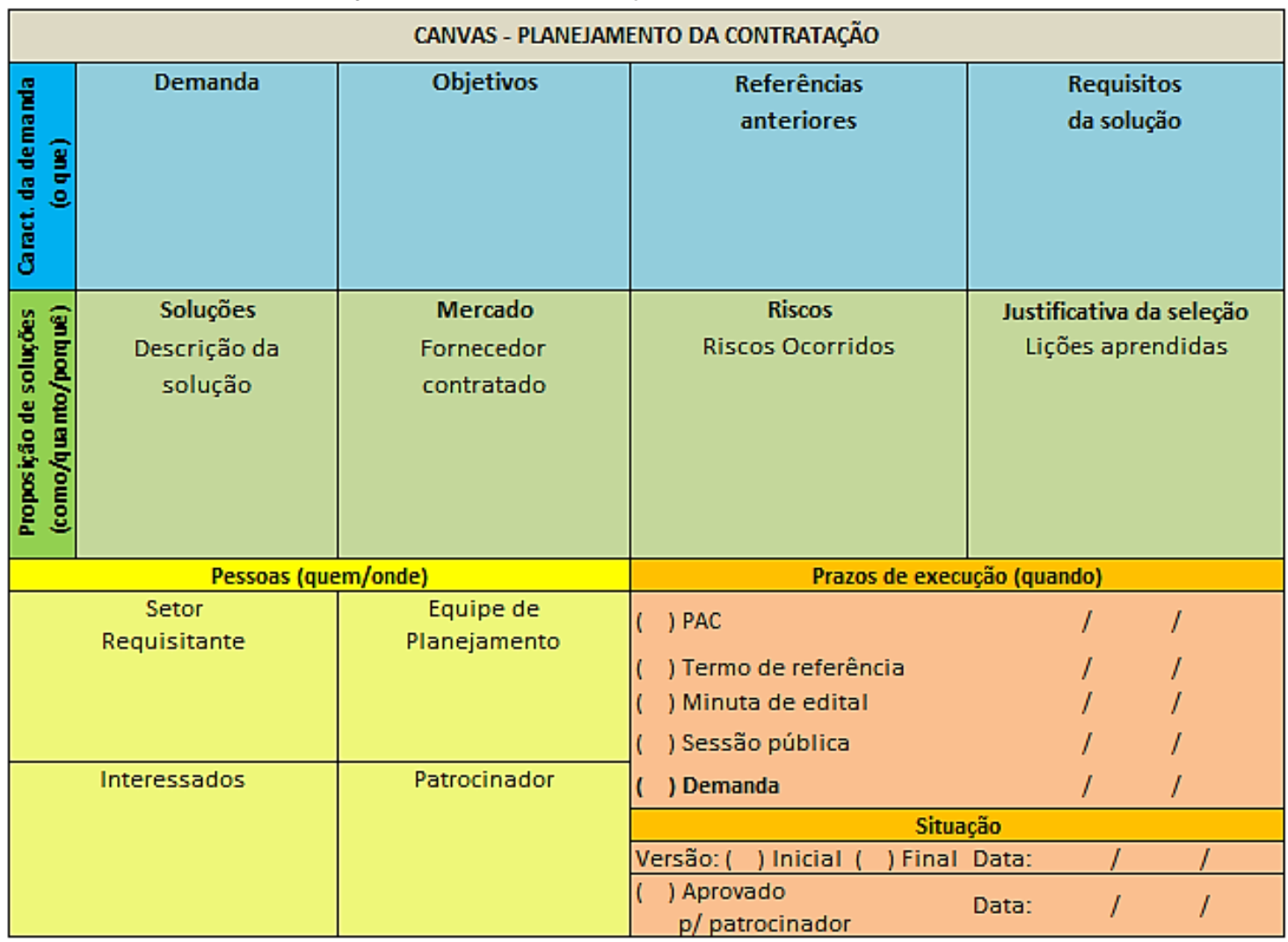

Fonte: Elaborado pelos autores (2019).

Conforme observado na Figura 1, a primeira linha é destinada à Caracterização da Demanda, cujo foco é responder o que se pretende adquirir. Nela constam os campos demanda, objetivos, referências anteriores e requisitos da solução. Já a segunda linha contempla os questionamentos como, quanto e o porquê, pormenorizados nos quadros soluções, mercado, riscos e justificativa da solução. As indagações quem e onde são traduzidas nos campos setor requisitante, equipe de planejamento, interessados e patrocinador. $\mathrm{O}$ quando se refere ao prazo para execução da demanda e sua correspondência com o PAC. Por último, o documento necessita ser aprovado pelo patrocinador.
No Canvas, os componentes da parte azul mantêm conexão com os itens da parte verde. Desse modo, para atender a demanda, há de se observar as soluções disponíveis no mercado; já os objetivos da contratação serão atingidos por meio dos serviços prestados pelo fornecedor (mercado); enquanto as referências anteriores possibilitam indicar os possíveis riscos inerentes à nova aquisição. Quanto aos requisitos da solução, critérios estabelecidos para aquisição do objeto, esses devem justificar a solução escolhida.

Como o artefato pretende responder aos questionamentos do DOC, ET e MR, observase a interligação entre os documentos, onde a finalização de um corresponde ao início do outro (Santana, 2015; Fenili, 2018). Ao se 
comparar a disposição dos elementos no referências estão alinhadas à IN 5/2017, Canvas, é possível constatar como as conforme demonstrado na Figura 2.

Figura 2- Alinhamento entre o Canvas e a IN 5/2017

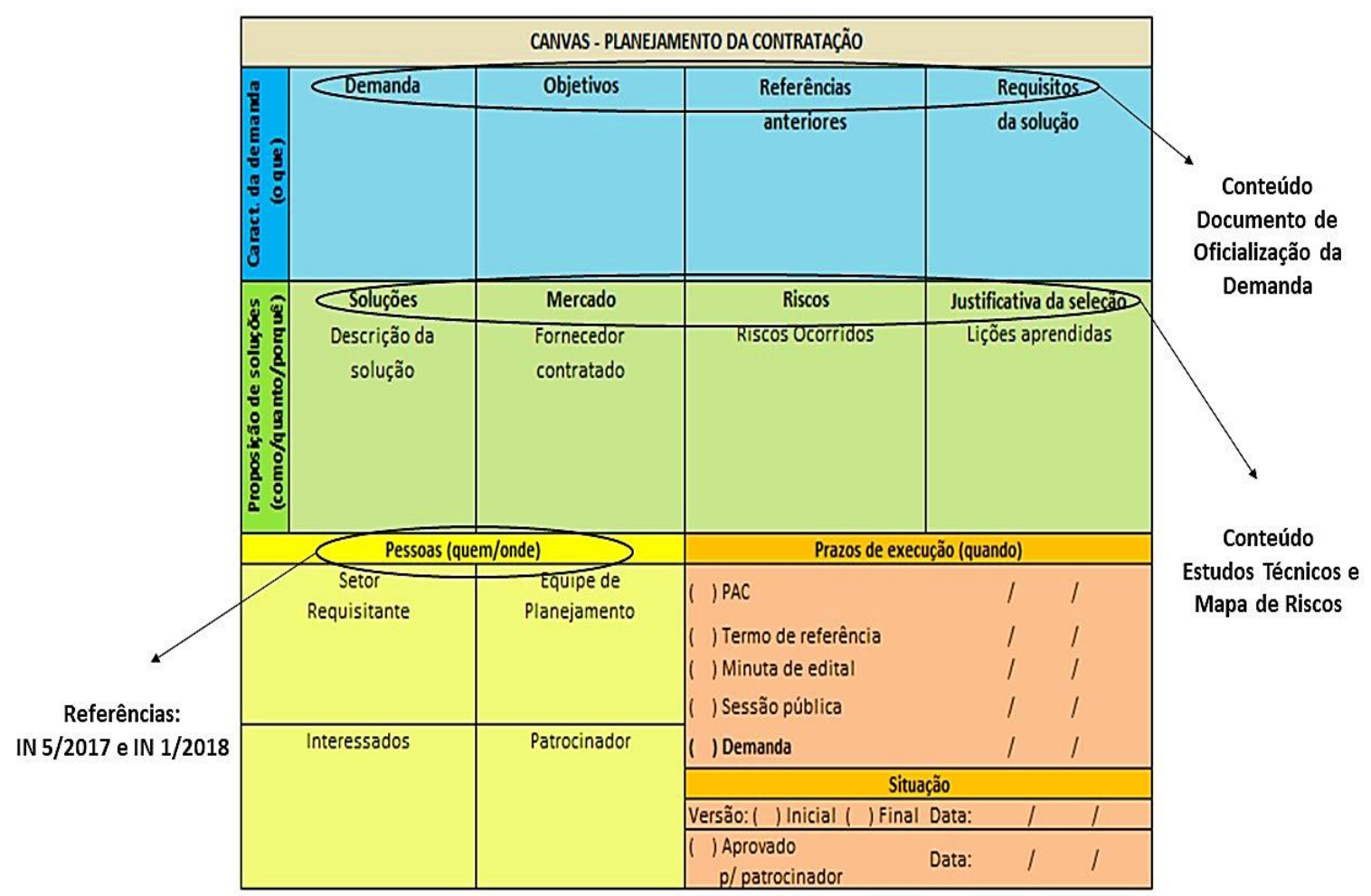

Fonte: Elaborado pelos autores (2019).

Além disso, as similaridades entre o Canvas e o LCC (Veras, 2016) são identificadas no layout, na divisão, na correspondência dos campos, nos objetivos e na sequência lógica dos requisitos. No entanto, apresenta limitações em virtude de sua aplicação ser destinada ao planejamento de compras, enquanto o LCC é aplicável a todos os tipos de projeto, durante todo o ciclo de vida (Veras, 2016; Medeiros, Araújo \& Oliveira, 2018). A Figura 3 ilustra a referida representação. 
Figura 3 - Relação entre o LCC e o Canvas do Planejamento

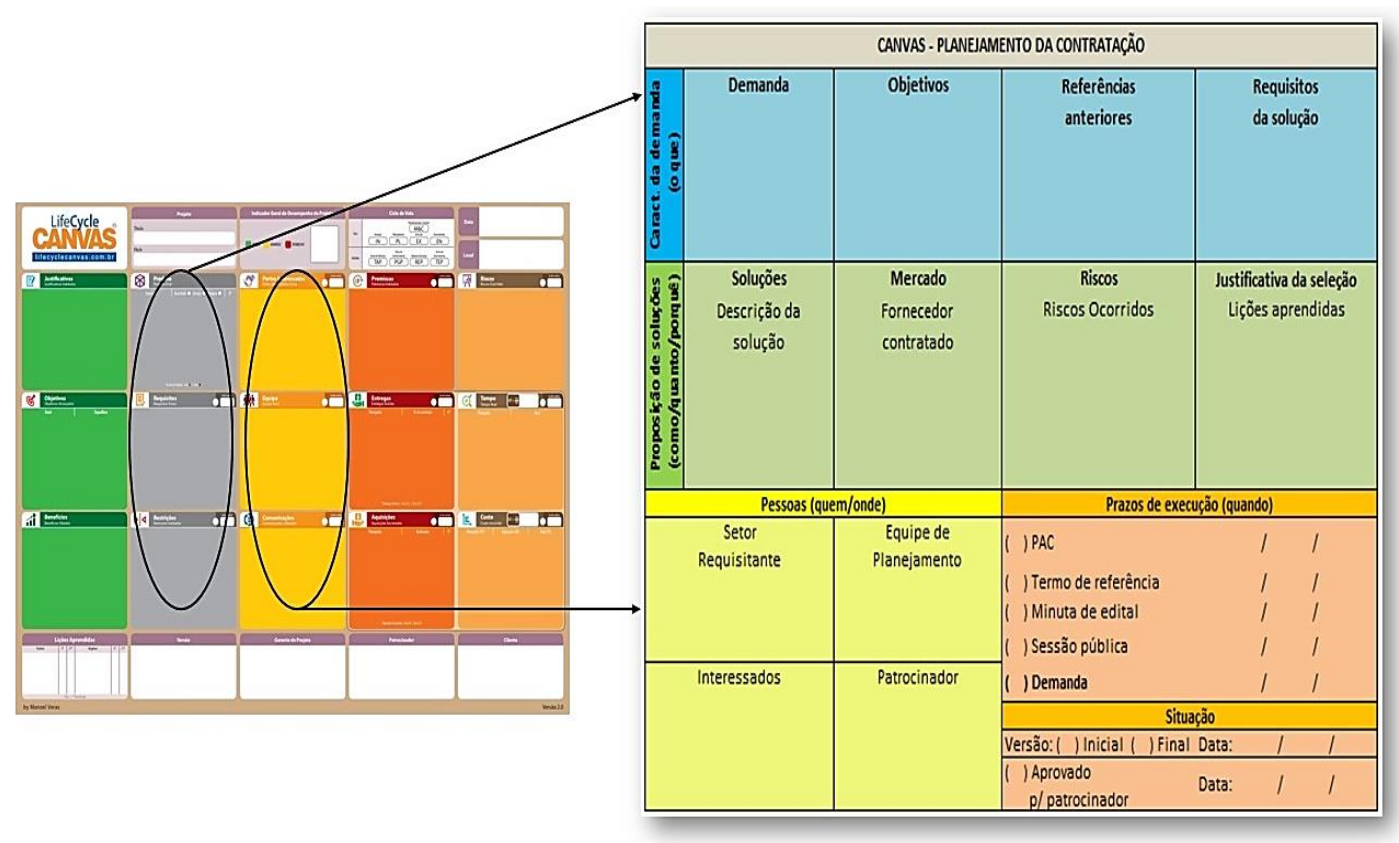

Fonte: Elaborado pelos autores (2019).

Após a exposição do Canvas como ferramenta auxiliar, a Figura 4 apresenta o "Framework para o Planejamento de Compras
Públicas”. Em seguida, serão descritas as orientações de como implementá-lo junto à equipe de planejamento contratação da IFES.

Figura 4 - Framework para o Planejamento de Compras Públicas

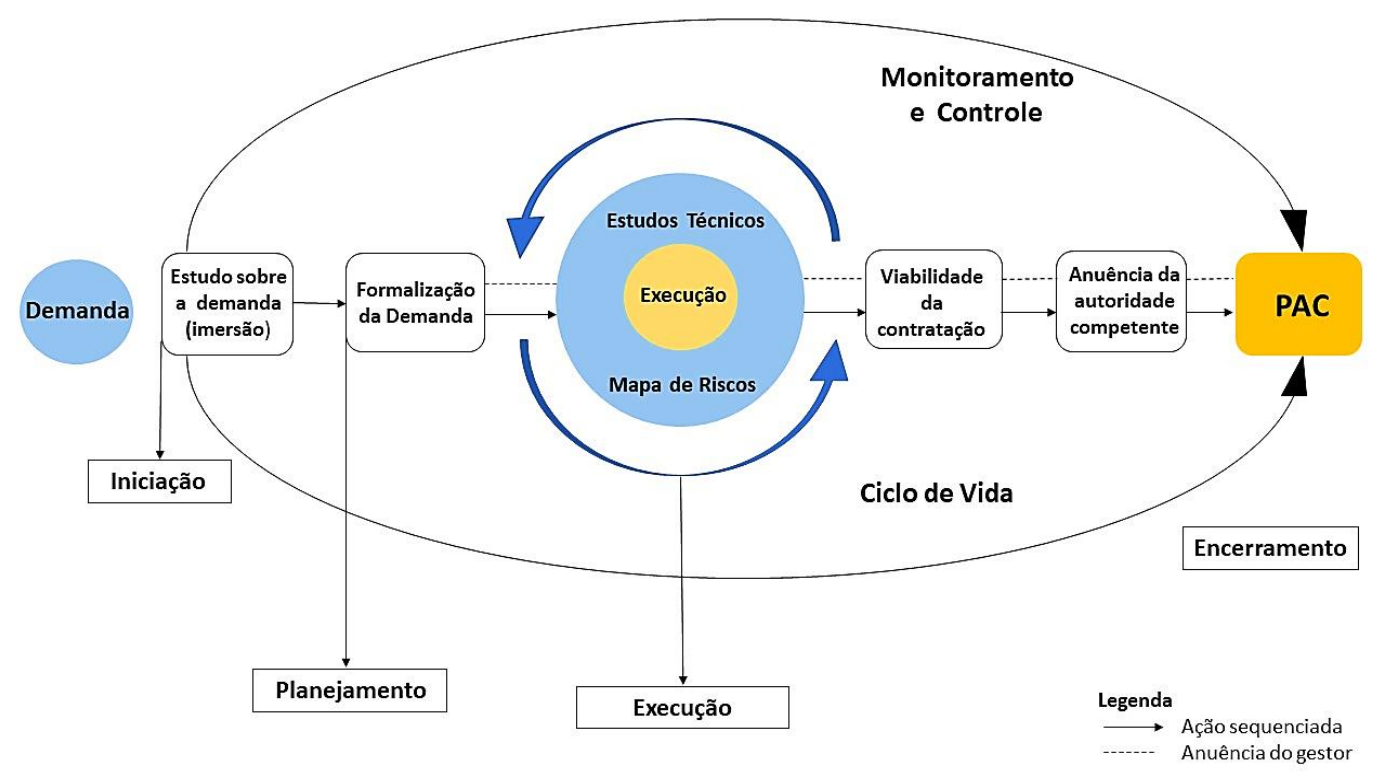

Fonte: Produto da pesquisa (2019). 
Para estabelecer a conexão entre o planejamento da contratação e os grupos de processos de gerenciamento, visto ser concebido como um projeto, foram utilizados os conceitos básicos dos trabalhos de Vargas (2009, 2018), Carvalho \& Rabechini Júnior (2017), Veras (2016), além das boas práticas recomendadas no $\mathrm{PMBOK}^{\circledR}$.

Este framework é constituído por uma metodologia híbrida, uma vez que adapta técnicas do gerenciamento tradicional de projetos a modelos visuais como canvas e quadro de tarefas eletrônico com a abordagem kanban, no contexto das universidades federais. Cabe esclarecer que embora seja oferecido um passo a passo para realização das tarefas, característica essa inerente aos frameworks (Deeb et al., 2018; Seifullina et al., 2018), o planejamento de compras não é uma atividade repetitiva. Dessa maneira, além das demandas serem variáveis, a escolha da equipe do projeto também pode influenciar nos resultados (Carvalho \& Nelson, 2020).

A base conceitual deste framework remete aos trabalhos de Veras (2016) e Vargas $(2009,2018)$ quanto aos grupos de processos de gerenciamento, que marcam as fases mas não correspondem diretamente ao ciclo de vida. Conforme sugere Veras (2016), nem todos os processos descritos nos grupos de gerenciamento do $\mathrm{PMBOK}^{\circledR}$ serão utilizados. Portanto, recorre-se aos que mantêm maior relevância ao atendimento das especificidades exigidas ao planejamento de compras públicas.
Quanto ao ciclo de vida, adota a perspectiva dos grupos de processos ou fases de gerenciamento do $\mathrm{PMBOK}^{\circledR}$, sendo elas: iniciação, planejamento, execução, monitoramento e controle e encerramento (PMI, 2017, Vargas, 2009,2018); e as fases sequenciadas propostas por Veras (2016).

Para o gerenciamento das etapas do ciclo de vida do projeto, utiliza recursos como Canvas, check-list em plataforma eletrônica de tarefas baseada no kanban, técnica de brainstorming, cronograma para reuniões presenciais; além de indicar os meios de comunicação à equipe do projeto. Também orienta que uma entrega só entrará em execução após a finalização da anterior, e vincula um tempo determinado para cada atividade.

A mudança de fase é marcada pela conclusão de uma entrega (Vargas, 2018). Desse modo, ao unir os grupos de processos de gerenciamento e o planejamento de compras públicas, tem-se as seguintes etapas: a Iniciação é marcada pelo preenchimento do Canvas (TAP); o Planejamento (quadro de tarefas elaborado); a Execução (entregas concluídas); o Monitoramento e Controle (permeia todo o ciclo de vida do projeto); e o Encerramento (validação de todas as entregas e elaboração do relatório final (TEP). Já o produto corresponde às informações do objeto a serem inseridas no PAC.

No que concerne aos papéis, os principais atores deste framework são: a) a equipe do projeto, composta, preferencialmente, pelos servidores da equipe de planejamento de 
compras; b) o facilitador, responsável por resolver os problemas e manter o contato entre o patrocinador e a equipe do projeto; e c) o patrocinador, representado pela chefia da unidade ou responsável pelo setor requisitante. Por força das instruções normativas, as entregas são previamente determinadas; enquanto a validação corresponde ao aval do patrocinador sobre a conclusão das atividades.

As inovações tecnológicas têm oferecido vários softwares e aplicativos para a gestão de projetos. Para o acompanhamento, optou-se por uma ferramenta gerencial eletrônica que utiliza a lógica do kanban (Medeiros, Gurgel \& Veras, 2017; Oliveira,
Oliveira, \& Michaloski, 2020), modelo visual correspondente às pretensões deste trabalho. Outro ponto considerado foi a recorrência do uso dessa ferramenta na instituição, além de disponibilizar uma versão gratuita.

Conforme expresso no capítulo Metodologia, os critérios estabelecidos para validação deste framework foram adaptação, participação, comunicação e contribuições práticas. A escolha desses quatro aspectos foi pautada na literatura existente sobre o tema, dividido por cada elemento e sua vertente teórica. A Tabela 2 os representa de forma sintetizada.

Tabela 2 - Critérios para validação do framework

\begin{tabular}{|c|c|c|}
\hline CRITÉRIOS & DESCRIÇÃO & AUTORES \\
\hline ADAPTAÇÃO & $\begin{array}{l}\text { Verificar a adaptação da equipe à } \\
\text { metodologia de trabalho proposta quanto ao } \\
\text { uso de ferramentas e aplicabilidade do } \\
\text { framework }\end{array}$ & $\begin{array}{l}\text { Glodzinski (2018); Deeb et al. (2018); } \\
\text { Almanei, Salonitis e Tsinopoulos } \\
\text { (2018) e Seifullina et al. (2018); } \\
\text { Fernandes (2019) }\end{array}$ \\
\hline PARTICIPAÇÃO & $\begin{array}{l}\text { Verificar a atuação da equipe por meio do } \\
\text { comprometimento com as entregas, os } \\
\text { prazos e presença nas reuniões. }\end{array}$ & $\begin{array}{l}\text { Luyet et al. (2012); Nascimento } \\
\text { (2018); Lindsjørn et al. (2016), Tjell e } \\
\text { Bosch-Sijtsema (2015); e Burger, } \\
\text { White \& Yearworth (2019) }\end{array}$ \\
\hline COMUNICAÇÃO & $\begin{array}{l}\text { Observar como as } \\
\text { comunicação escolhidas } \\
\text { conclusão do projeto. }\end{array}$ & $\begin{array}{l}\text { Carvalho e Rabechini Jr. (2017); } \\
\text { Vargas (2009, 2016); Veras (2016); } \\
\text { PMI (2017); Oliveira, Oliveira \& } \\
\text { Michaloski (2020) }\end{array}$ \\
\hline $\begin{array}{l}\text { CONTRIBUIÇÕES } \\
\text { PRÁTICAS }\end{array}$ & $\begin{array}{l}\text { Analisar a contribuição efetiva para } \\
\text { elaboração dos documentos exigidos para o } \\
\text { planejamento da contratação, com } \\
\text { observância aos prazos estipulados. }\end{array}$ & $\begin{array}{l}\text { Vargas (2009, 2016); Veras (2016), } \\
\text { PMI (2017); Fenili (2018); Santana } \\
\text { (2015); Yeow e Edler (2012); Szalay, } \\
\text { Kovács e Sebestyén (2017); Deeb et } \\
\text { al. (2018); Carvalho \& Nelson (2020) }\end{array}$ \\
\hline
\end{tabular}

Fonte: Elaborado pelos autores (2019).

Os requisitos de qualidade do projeto (Carvalho \& Rabechini Júnior, 2017; Vargas, 2018) estão mais atrelados ao que apregoam os normativos de compras públicas. Desse modo, devem ser atendidos por meio de documentos bem fundamentados, cuja pesquisa de preços obedeça aos critérios legais. Esses elementos fomentam uma consistente análise das soluções 
disponíveis no mercado para se escolher a mais viável (Carvalho \& Nelson, 2020).

No tocante à aplicação do framework, esta ocorreu entre os meses de dezembro de 2018 e janeiro de 2019 , e buscou respeitar os prazos estabelecidos no calendário de compras institucional. Por seu turno, o objetivo do projeto consistiu em elaborar de forma satisfatória os documentos exigidos no PAC. Quanto à sua justificativa, correspondeu a desenvolver estratégicas que possibilitassem pensar e planejar melhor a solução contratada.

Cabe ressaltar que a aplicação junto à equipe de planejamento foi realizada como um projeto piloto (Seifullina et al.,2018), o que permitiu realizar ajustes; assim como ocorreu em tempo reduzido e adequado à disponibilidade dos participantes (Soares et $a l ., 2019)$.

Os dados apontam que a equipe se adaptou à metodologia proposta, com destaque para utilização do Canvas e da ferramenta do quadro visual eletrônico. Foi participativa, compareceu a todas as reuniões presenciais e colaborou para conclusão das entregas; utilizou recursos como mensagens instantâneas e correio eletrônico para se comunicar e trocar informações. Por fim, o framework demonstrou sua contribuição prática, uma vez que os documentos que integravam o PAC foram entregues conforme o previsto.

No que concerne ao sucesso do projeto, Vargas (2009) indica que este ocorre quando é executado de acordo com o que foi planejado. De forma ampla, diz-se que o projeto alcançou um bom desempenho quando concluído respeitando o prazo (PMI, 2017), o custo e os objetivos planejados (Vargas, 2018).

No que diz respeito ao sucesso do projeto no ambiente organizacional, Vargas (2018) indica que acontece por meio da realização dos benefícios previstos e quando as saídas se transformam em resultados para atender os objetivos estratégicos da organização (Campos et al.,2020; Oliveira, Oliveira, \& Michaloski, 2020). Nesse sentido, o cronograma foi cumprido, as reuniões realizadas conforme o programado, $\mathrm{e}$ as entregas concluídas atendendo aos padrões de qualidade exigidos em cada documento (DOD, ET e MR).

Tanto o Canvas quanto as entregas foram aprovados pelo patrocinador. Além disso, a realização das lições aprendidas (Veronese, 2014; Chaves et al., 2016; Duffield \& Whitty, 2016) e a elaboração do relatório final coadunam com as boas práticas descritas no PMBOK $^{\circledR}$.

A partir dos trechos extraídos dos áudios, foi possível inferir que, como indicam os trabalhos de Veras (2016), Barbosa et al. (2018) e Oliveira, Oliveira, \& Michaloski (2020), os modelos visuais do tipo canvas facilitam o gerenciamento e compartilhamento da comunicação entre os envolvidos, uma vez que os dados do projeto ficaram visíveis a todos os membros da equipe (Tjell \& Bosch-Sijtsema, 2015; Lindsjørn et al.,2016).

Por sua vez, considerar a percepção dos profissionais (Glodzinski, 2018), o fator 
humano (Deeb et al.,2018), o alinhamento entre a estratégica do negócio e as metas e objetivos dos colaboradores (Almanei, Salonitis \& Tsinopoulos, 2018) é importante em um cenário de mudanças, visto que elas impactam não somente processos, mas pessoas (Szalay, Kovács \& Sebestyén, 2017).

Glodzinski (2018) aponta que utilizar a expertise dos colaboradores durante a implementação de novos procedimentos e processos de trabalho pode levar a obtenção de melhores resultados (Deeb et al.,2018). Os dados também se interligam aos estudos de Szalay, Kovács e Sebestyén (2017), que defendem a junção entre a teoria e prática organizacional para implementação de novas ideias, aliada à constante capacitação da equipe (Nascimento \& Santos, 2018; Seifullina et al., 2018). Logo, infere-se que os participantes utilizaram sua vivência cotidiana e conhecimento técnico para melhor instruir o planejamento de compras, proporcionando, assim, mais qualidade aos documentos elaborados.

Por outro lado, para três dos quatro participantes, o fator tempo foi avaliado como um ponto negativo. Isto corrobora o trabalho de Nascimento (2018), que ao estudar o fenômeno do engajamento em equipes de projetos no setor público, expôs a dificuldade de os integrantes conciliarem suas atividades funcionais com as demandas do projeto (Carvalho \& Nelson, 2020).

Apesar dessa limitação, Nascimento (2018) destacou como pontos positivos o reconhecimento do trabalho e a coesão do grupo. Dessa maneira, os pesquisadores endossam a sugestão do autor para regulamentar, em nível institucional, a participação dos servidores em projetos organizacionais, de modo a ser possível dedicarem algumas horas de trabalho exclusivamente às atividades da iniciativa.

No que concerne ao registro das informações obtidas por meio do projeto de contratação, Chaves et al. (2016) e Duffield \& Whitty (2016) discorrem que mesmo quando as organizações dispõem da estrutura adequada à aprendizagem, não é comum as equipes de projetos aplicarem esse conhecimento em eventos posteriores. Isto porque as lições aprendidas recebem pouca atenção nas pesquisas referentes ao gerenciamento de projetos (Veronese, 2014; Chaves et al.,2016).

A partir dessa constatação, os pesquisadores sugerem a criação de um repositório institucional para armazenar os relatórios finais de cada projeto de planejamento de compras, com a finalidade de oferecer suporte a produções semelhantes futuramente. Tal ação poderá contribuir para a tendência de inovações em compras públicas, consideradas como um sistema dinâmico e colaborativo (Santana, 2015; Ferrer, 2015; Fenili, 2018; Fernandes, 2019).

\section{Considerações finais}

Em tempos de contingenciamento de despesas, as discussões sobre o gasto público, a eficiência e a qualidade dos serviços oferecidos 
à população suscitam a necessidade de setor público reavaliar práticas, mensurar desempenho, assim como desenvolver metodologias e ferramentas inovadoras capazes de otimizar os limitados recursos.

Nesse cenário, as universidades federais desempenham um relevante papel, não apenas por sua missão social de formar cidadãos e qualificá-los para o mercado de trabalho, mas como compradores públicos. Isto porque as IFES destinam considerável parcela dos seus recursos financeiros à contratação de bens e serviços, os quais dão suporte às atividades finalísticas de pesquisa, ensino e extensão.

Por um lado, o efetivo planejamento das compras institucionais resulta em melhores aquisições e contratos que impactam positivamente na qualidade dos serviços oferecidos à comunidade universitária. Por outro, falhas significativas nessa fase originam a indisponibilidade de itens licitados, comprometendo, assim, a continuidade das ações acadêmicas e administrativas.

Diante disso, o objetivo deste estudo foi desenvolver um framework para o planejamento de compras públicas, o qual é concebido como um projeto, integrando os preceitos das instruções normativa IN 5/2017 e IN 1/2018 aos modelos visuais tipo canvas, reconhecidos por facilitarem o gerenciamento e melhorarem a comunicação entre a equipe do projeto.

A metodologia sistematizada do framework utiliza ferramentas visuais como Canvas do Planejamento da Contratação e o quadro de tarefas eletrônico para o acompanhamento e controle; estabelece um cronograma de reuniões e tempo determinado para cada tarefa; e orienta a equipe do projeto sobre a elaboração dos documentos que devem ser inseridos no PAC.

Após a aplicação e a validação do modelo, os resultados indicam que esses elementos tornaram a atuação da equipe mais dinâmica e contribuíram para que as entregas fossem concluídas conforme escopo, prazos e critérios planejados. Como aspectos negativos, os participantes destacaram o pouco tempo para realização das atividades, bem como a dificuldade em conciliar as atribuições do projeto com as respectivas funções laborais.

Os dados obtidos nesta pesquisa colaboram com a literatura à medida que oferecem uma nova perspectiva ao planejamento das compras públicas. Por sua aplicação prática, auxilia o agente de compras a pensar e avaliar as soluções disponíveis no mercado fornecedor, monitorar os processos de forma dinâmica, promover o engajamento e comunicação das equipes de trabalho. Juntos, esses componentes contribuem para qualidade dos estudos técnicos e termos de referência, documentos que conjugam as especificações e requisitos dos objetos a serem contratados, e são imprescindíveis para o sucesso da licitação.

Como limitações, aponta-se o fato de o Framework para o Planejamento de Compras Públicas ter sido aplicado apenas a uma equipe de planejamento da contratação da IFES; além de focar somente em um objeto (do tipo prestação de serviços), dentre as muitas 
demandas a serem licitadas pela instituição.

Desse modo, não foi possível comparar a sua efetividade considerando a variedade e a complexidade de todos os itens do catálogo.

No que diz respeito à formação de agenda para trabalhos futuros, sugere-se aplicar de forma mais sistematizada o framework a outras equipes de planejamento de compras na IFES, para confrontar os dados obtidos nesse primeiro teste. Outro aspecto é avaliar a introdução de novas técnicas e ferramentas utilizadas no gerenciamento de projeto, como os métodos ágeis.

\section{Referências}

Almanei, M., Salonitis, K. \& Tsinopoulos, C. (2018). A conceptual lean implementation framework based on change management theory. 51st CIRP Conference on Manufacturing Systems. Procedia CIRP 72, p.1160-1165.

Araújo, S. S de, Matos, L. dos S. \& Ensslin, S. R. (2020) Compras Públicas Sob a Perspectiva da Avaliação de Desempenho: Uma Revisão de Literatura e Agenda de Pesquisa. Revista Gestão \& Conexões Management and Connections Journal, Vitória (ES), v. 9, n. 1, jan./abr. 2020.p.99127.

Avigo, R. O., \& João-Roland, I. de S. (2019). Inovação organizacional em compras públicas pelo SRP: análise da acurácia do planejamento participativo do Instituto Federal do Triângulo Mineiro (IFTM). Revista GUAL, Florianópolis, v. 12, n. 3, p. 173-195, setembro-dezembro 2019.

Bittencourt, S. (2015). Licitações para contratação de serviços continuados ou não: a terceirização na administração pública. São Paulo: Matrix.

Brasil. Ministério do Planejamento, Orçamento e Gestão (2008). Instrução Normativa $n^{o} 2$. Dispõe sobre regras e diretrizes para contratação de serviços, continuados ou não.
Brasil. Secretaria de Logística e Tecnologia da Informação (2008) Instrução Normativa $n^{\circ} 4$, de 19 de maio de 2008.

Brasil. Ministério do Planejamento, Desenvolvimento e Gestão (2017). Instrução normativa $n^{\circ} 5$. Dispõe sobre as regras e diretrizes do procedimento de contratação de serviços.

Brasil. Ministério do Planejamento, Desenvolvimento e Gestão (2018). Instrução Normativa $n^{o} 1$. Dispõe sobre o Sistema de Planejamento e Gerenciamento das Contratações e sobre a elaboração do Plano Anual de Contratações.

Brasil. Câmara dos Deputados. Orçamento da União.

Barbosa, F. J. M. et al. (2018) Visualização da informação e métodos visuais como ferramentas estratégicas para o gerenciamento de projetos Revista de Gestão e Projeto-GeP, vol.9, N.1. Jan/abr, p.102114. e-ISSN: 2236-0972 DOI: 10.5585/gep.v9i1.635

Burger, K., White, L., \& Yearworth, M. (2019). Understanding front-end project workshops with Social Practice Theory. International Journal of Project Management, v. 37, p.161-175. doi.org/10.1016/j.ijproman.2018.11.003.

Campos, M. C. et al (2020). Avaliação de maturidade em gestão de projetos na Universidade Federal de Alagoas utilizando o Método Prado-MMGP. Revista de Gestão e Projetos (GeP), 11(1), 01- 16. https://doi.org/10.5585/gep.v11i1.15870.

Carvalho, M.C.S., \& Nelson, A.V.M (2020). Gerenciamento de projetos: um modelo de planejamento para licitações de serviços no Instituto Federal de Sergipe - Campus Aracaju. IN: ARAÚJO, Maria Arlete de; ARAÚJO, Fábio Resende de (org.). Compras na administração pública: processos, transparência e sustentabilidade em aquisições e contratações [recurso eletrônico] Natal: EDUFRN, 2020. 2 v. ISBN 978-8542509-49-6.

Carvalho, M. M., \& Rabechini Jr. R. (2017). Fundamentos em gestão de projetos: construindo competências para gerenciar projetos. 4.ed. São Paulo: Atlas, 2017.

Chaves, M. S. et al. (2016) A new approach to managing Lessons Learned in PMBoK process groups: the Ballistic 2.0 Model. 
International Journal of Information Systems and Project Management, vol.4, n.1, p. 2745. doi: 10.12821/ijispm040102.

Costa, A. F., Braga, C. R. A., \& Andrioli, L. G. G. (2017). Estudo técnicos preliminares: o calcanhar de Aquiles das aquisições públicas. Revista do TCU, ano 49, número 139, p.3851.

Deeb, S. et al. (2018). A generic framework to support the implementation of six sigma approach in SMEs. IFAC Paper Online Conference Paper Archive, 51-11, p. 921 926.

Duffield, S. M., \& Whitty, S. J. (2016). Application of the Systemic Lessons Learned Knowledge model for organizational learning through projects. International Journal of Project Management, vol.34, p. 1280 - 1293. doi.org/10.1016/j.ijproman.2016.07.001

Eaidgah, Y. et al. (2016). Visual management, performance management and continuous improvement: a lean manufacturing approach. International Journal of Lean Six Sigma, v.7, n. 2, p. 187-210. doi.org/10.1108/IJLSS-09-2014-0028

Fenili, R. (2018). Governança em aquisições públicas: teoria e prática à luz da realidade sociológica. Niterói/RJ: Impetus.

Fernandes, C. C. C. (2019). Compras Públicas no Brasil: tendências de inovação, avanços e dificuldades no período recente. Administração Pública e Gestão Social, 2019, 11(4), Setembro-Dezembro, ISSN: 2175-5787.

Ferrer, F. (2015). Os desafios do setor público para construir um novo modelo de abastecimento e compras: inovação ou estagnação. In: FERRER, Florencia; SANTANA, Jair Eduardo (coord). Compras públicas Brasil. 1.ed. Rio de Janeiro: Elsevier.

Flick, U. (2013). Introdução à metodologia de pesquisa: um guia para iniciantes. Porto Alegre: Penso.

Finocchio Júnior, J. (2013). Project Model Canvas: gerenciamento de projetos sem burocracia. Rio de Janeiro: Elsevier/Campus.

Fuentes-Bargues, J.L, Ferrer-Gisbert, P.S, \& González-Cruz, M.C. Analysis of Green Public Procurement of Works by Spanish Public Universities (2018). International Journal of Environmental Research and
Public Health 2018, 15, 1888; doi:10.3390/ijerph15091888.

Glodzinski, E. (2018). Project assessment framework: multidimensional efficiency approach applicable for project-driven organizations. Conference on Health and Social Care Information Systems and Technologies, Procedia Computer Science, v. 138, p. $731-738$.

10.1016/j.procs.2018.10.096

Justen Filho, M. (2016). Curso de direito administrativo. 12.ed. rev., atual. e ampl. São Paulo: Editora Revista dos Tribunais.

Kerzner, H. (2006). Gestão de projetos: as melhores práticas. 2.ed. Porto Alegre: Bookman.

Lindsjørn, Y. et al. (2016). Teamwork quality and project success in software development: a survey of agile development teams. The Journal of Systems and Softwares, 122, p. 274 - 286. doi.org/10.1016/j.jss.2016.09.028

Link, C. P., Silva, G., Barichello, R., \& Dal Magro, C. B. (2020). Fatores críticos no gerenciamento de projetos públicos sustentáveis. Revista de Gestão e Projetos (GeP), 11(2), 87-109. doi.org/10.5585/gep.v11i2.17490

Luyet, V. et al. (2012) A framework to implement stakeholder participation in environmental projects. Journal of Environmental Management, v.111, 2012, p. $213-219$. http://dx.doi.org/10.1016/j.jenvman.2012.06. 026

Matias-Pereira, J. (2012). Curso de gestão estratégica na administração pública. São Paulo: Atlas.

Medeiros, B. C. (2017). Life Cycle Canvas (LCC): análise de um modelo de gestão visual para o planejamento de projetos. 2017. 207f. Tese (Doutorado em Administração) - Universidade Federal do Rio Grande do Norte, Natal/RN.

Medeiros, B. C., Araújo, V. F. Si., \& Oliveira, M. K. A. S. (2018). Life Cycle Canvas (LCC): um modelo visual para a gestão do ciclo de vida do projeto. Revista de Gestão e Projetos - GeP, v.9, n.1, p. $87-101$. doi.org/10.5585/gep.v9i1.628

Medeiros, J. V., Gurgel, A. M., \& Veras, M. (2017) Proposta de uma abordagem em gestão de projetos baseada no LifecycleCanvas e Kanban. In: VI SINGEP - 
Simpósio Internacional de Gestão de Projetos, Inovação e Sustentabilidade, São Paulo. Anais [...]

Nascimento, L. D. P. (2018). Engajamento em equipes de projetos à luz da teoria JR-D. 2018. 167f. Dissertação (Mestrado em Gestão de Processos Institucionais) Universidade Federal do Rio Grande do Norte, Natal/RN.

Nascimento, R.N. O., \& Santos, L. G. (2018). Gestão de compras no serviço público: um estudo de caso em uma universidade federal potiguar. EmpíricaBR - Revista Brasileira de Gestão, Negócio e Tecnologia da Informação. 2018, 229-249.

Neves, M.Y., \& Moré, R.P.O. (2020) Pregão eletrônico: um estudo das causas de cancelamento de itens no âmbito de uma universidade federal. Revista do Serviço Público (RSP), Brasília 71 (1) 171-205 jan/mar 2020.

https://doi.org/10.21874/rsp.v71i1.3937| ISSN: 0034-9240 | E- ISSN: 2357-8017.

Oliveira, R. A. de, Zych, D. R., Oliveira, J. de, \& Michaloski, A. O. (2020). Desafios no uso de metodologias ágeis de gestão de projetos em órgãos públicos: um estudo de caso da Receita Estadual do Paraná. Revista de Gestão e Projetos (GeP), 11(2), 12-36. https://doi.org/10.5585/gep.v11i2.16522

Prodanov, C. C., \& Freitas, E. C. (2013). Metodologia do trabalho científico [recurso eletrônico]: métodos e técnicas da pesquisa e do trabalho acadêmico. 2.ed. Novo Hamburgo: Feevale.

PMI - Project Management Institute (2017). Um guia do conhecimento de projetos. Guia $\mathrm{PMBOK}^{\circledR}$. 6.ed., PMI.

Quadros, A. S., \& Carvalho, H. G. (2012) $O$ gerenciamento da comunicação de projetos públicos: como adaptar os processos do PMBOK/PMI à realidade da administração pública. Revista Brasileira de Planejamento e Desenvolvimento, v. 1, n. 1, p. 52-60. 10.3895/rbpd.v1n1.3097

Ramírez-Gutiérrez, Z., Barrachina-Parlance, M., \& Ripoll-Feliu, V. Efficiency in higher education. Empirical study in public universities of Colombia and Spain (2020). BRAZILIAN JOURNAL OF PUBLIC ADMINISTRATION | Rio de Janeiro 54(3):468-500, May - June 2020. http://dx.doi.org/10.1590/0034761220190232x.

Sampieri, H., Collado, F., \& Lucio, B. (2013). Metodologia de pesquisa [recurso eletrônico]. 5.ed. Porto Alegre: Penso.

Santana, J. E. (2015). Planejamento nas licitações e contratações governamentais: estratégias para suprimentos públicos. Curitiba/PR: Editora Negócios Públicos.

Santana, J. E., Camarão, T., \& Chrispim, A. C. D. (2016). Termo de Referência: o impacto da especialização do objeto e do termo de referência na eficácia das licitações e contratos. 5.ed. Belo Horizonte: Fórum.

Seifullina, A. et al. (2018). A lean implementation framework for mining industry. IFAC Paper Online - Conference Paper Archive, 51-11, p. 1149 - 1154. doi.org/10.1016/j.ifacol.2018.08.435

Soares, A. R. et al (2019). O processo de compras e o desafio da etapa do planejamento: o caso da Universidade Federal Rural de Pernambuco. Navus, Florianópolis/SC, v. 9, n. 4, p. 264-273 out./dez. ISSN 2237-4558.

Szalay, I., Kovács, Á., \& Sebestyén, Z. (2017). Integrated Framework for Project Management Office Evaluation. Creative Construction Conference 2017, CCC 2017, 19-22 June 2017, Primosten, Croatia. Procedia Engineering, v.196, p. 578 - 584. doi.org/10.1016/j.proeng.2017.08.033

Tjella, J., \& Bosch-Sijtsema, P. M. (2015). Visual management in mid-sized construction design projects. Procedia Economics and Finance - $8^{\text {th }}$ Nordic Conference on Construction Economics and Organization, v.21, p.193 - 200. $10.1016 / \mathrm{S} 2212-5671(15) 00167-7$

UFRN - Universidade Federal do Rio Grande Do Norte. Portal da UFRN.

Vargas, R. (2009). Gerenciamento de projetos: estabelecendo diferenciais competitivos. 7.ed. Rio de Janeiro: Brasport.

Vargas, R. (2018). Manual prático do plano do projeto: utilizando o PMBOK Guide. 6.ed. Rio de Janeiro: Brasport.

Veras, M. (2016). Gestão Dinâmica de Projetos: Life Cycle Canvas ${ }^{\circledR}$. Rio de Janeiro: Brasport.

Veras, M., \& M, B. C. (2016). Life Cycle Canvas: gestão dinâmica de projetos. Mundo Project Management, v. 12, n. 70, p. 76. 
Veronese, G. S. (2014). Métodos para captura de lições aprendidas: em direção a melhoria contínua na gestão de projetos. Revista de Gestão e Projetos - GeP, v.5, n.1, p. $71-83$. doi.org/10.5585/gep.v5i1.250

Yeow, J., \& Edler, J. (2012). Innovation procurement as project. Journal of Public Procurement, Vol.12, n. 4, p.488-520. 10.1108/JOPP-12-04-2012-B002

Yin, R. K. (2001). Estudo de caso: planejamento e métodos. 2.ed. Porto Alegre: Bookman. 